\title{
Blinker Operation System Using Voice Recognition and Image Processing
}

\author{
Ryokan Tashiro $^{\mathrm{a}}$, Seiichi Serikawa ${ }^{\mathrm{b}}$, Yuhki Kitazono ${ }^{\mathrm{a},{ }^{*}}$

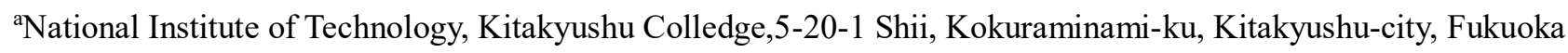 \\ 802-0985, Japan \\ ${ }^{b}$ Kyushu Institute of Technology, 1-1 Sensui-cho Tobata-ku Kitakyushu-city, Fukuoka 804-8550, Japan \\ *Corresponding Author: kitazono@kct.ac.jp
}

\begin{abstract}
Driving a car is difficult for people with physical disabilities. In particular, people with hand or fingertips turn blinkers on and off with their feet. However, there is a problem that customized cars are expensive. If people already have a car, it is expected that new purchase will not be realistic, as it is expected that the number of persons with physical disability license will increase with the aging of society. Therefore, we develop a system that such people can easily turn on blinker and hazard. This system is a system that emits blinkers and hazards in the face direction after being activated by voice. This has enabled people with hand and fingertip disabilities to turn on blinker and hazard while driving.
\end{abstract}

Keywords: blinker, voice recognition, servo motor

\section{Introduction}

According to the 2016 edition of the disabled person white paper of the Cabinet Office. Physically disabled person is 3937,000 and it is $3.1 \%$ of the total population ${ }^{(1)}$. Also, according to driver's license statistics of the Metropolitan Police Department's, in2017, the number of conditional driver's licenses for disabled persons is 40,499 cases only for the use of hearing aids, 196,364 cases as long as use of physically disabled vehicle and 3,912 cases of artificial hand, artificial leg and brace condition ${ }^{(2)}$. This number is less than $1 \%$ of the driver's license owners. As such, it is very difficult for disabled people to drive a car. Currently, rehabilitation hands and visual art interfaces that draw pictures using virtual brushes are being developed for people with such disabilities $^{(3)(4)}$. In addition, Japan is promoting the introduction of specially designed vehicles for disabled persons at driving schools. But because the content of the obstacle is various, it can't cope only with a specific vehicle. And the instructor who can understand the contents of disability and can teach individually is necessary. Also, there is the problem that customized cars are expensive. In addition, as it is expected that the number of persons with physical disability license will increase with the aging of society, new purchase is not realistic for people who already have cars. Thus, the current situation is that supply has not

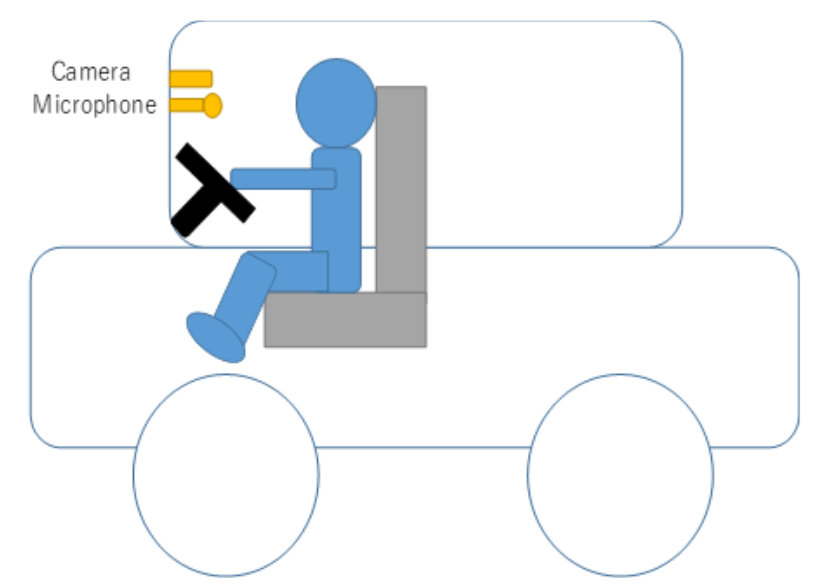

Fig.1. Diagram of the system

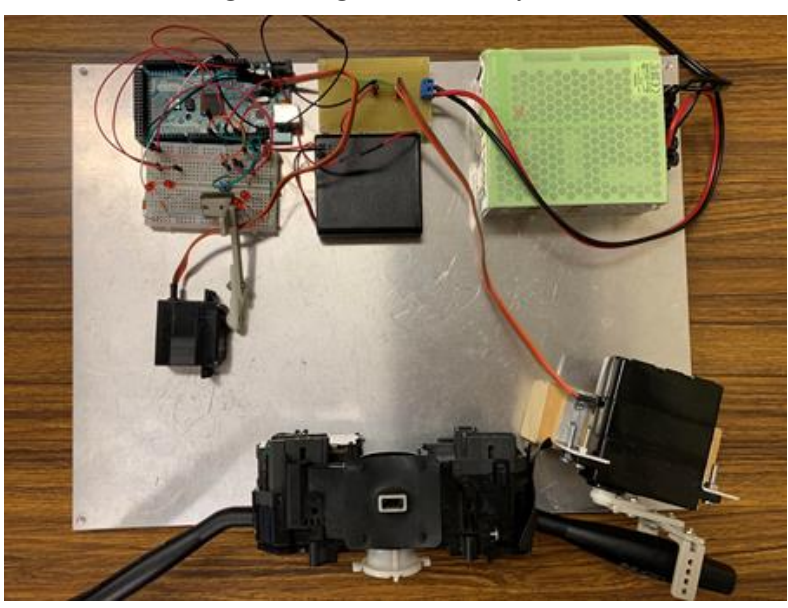

Fig.2. Overall view of the system 


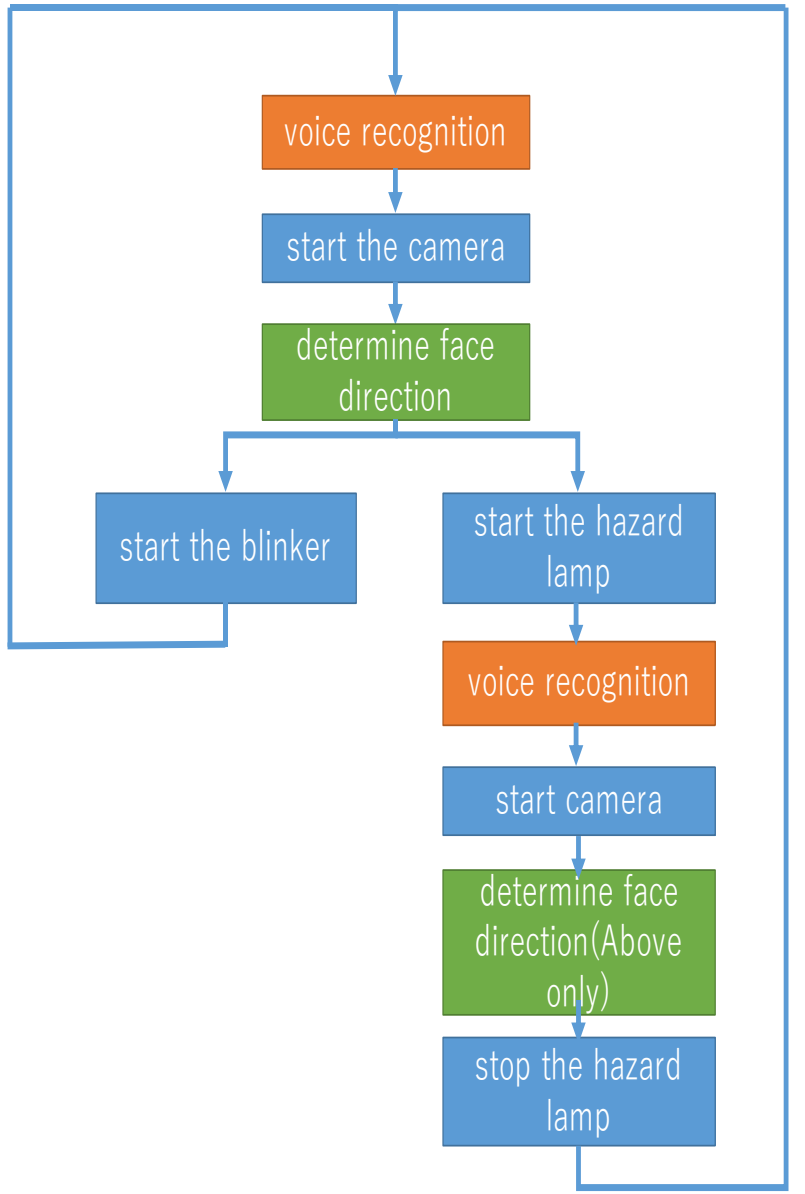

Fig.3. System flow

kept pace with demand. In this paper, we particularly focused on people with impaired arms and hands. Among such people, there are also people who drive using steering wheel with the right hand and drive using the special specification car that operates the accelerator and brake with the left hand. However, in this case, it is difficult to take out the blinker while driving, and there is a risk that the communication between the cars, the pedestrians, and the bicycles can't be sufficiently achieved, leading to an accident. Therefore, in this study, we propose a driving support system for people with hand and finger disabilities. As a similar study, there are blinkers for bicycles, which were developed for disabled people with arms or fingers to use face direction, but the study uses Kinect ${ }^{(5)}$. There is a problem that the cost is high. This system was created by Arduino, a camera, a microphone, and an operating unit. Speech recognition changed from Julius to "Voice recognition launcher F". We used voice recognition launcher $\mathrm{F}$ because it is easy to recognize from word registration and recognition rate is high. Moreover, since this assumes attaching to all cars, it is also possible to drive with a healthy person attached without having to buy a newly customized car.

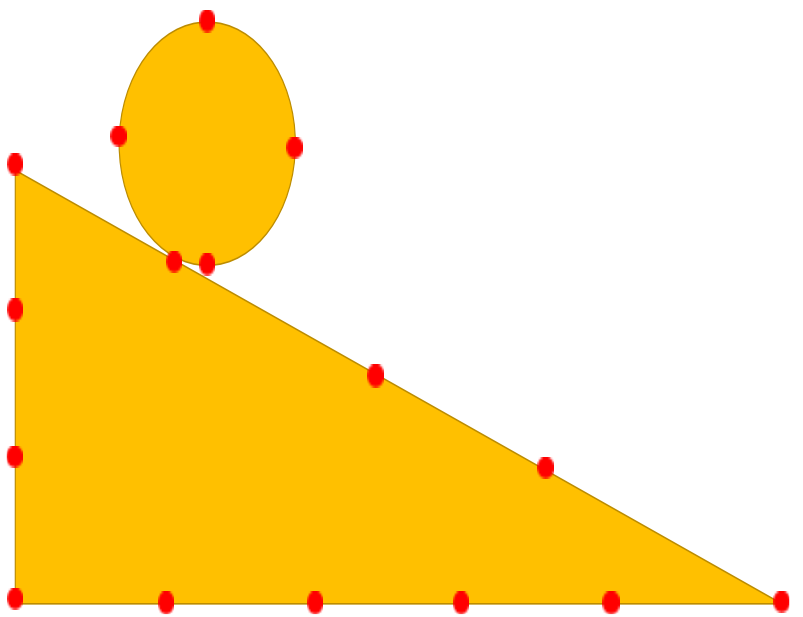

(a) initial position

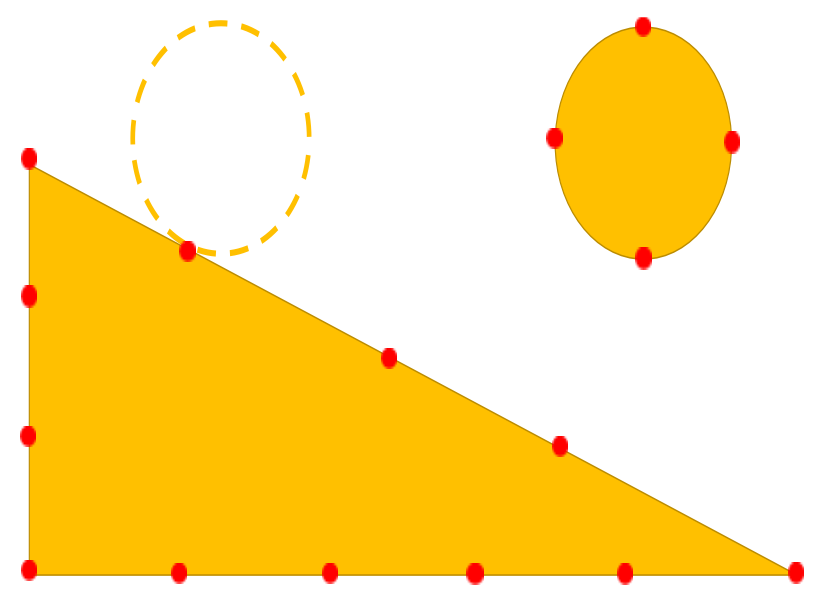

(b) Feature points move

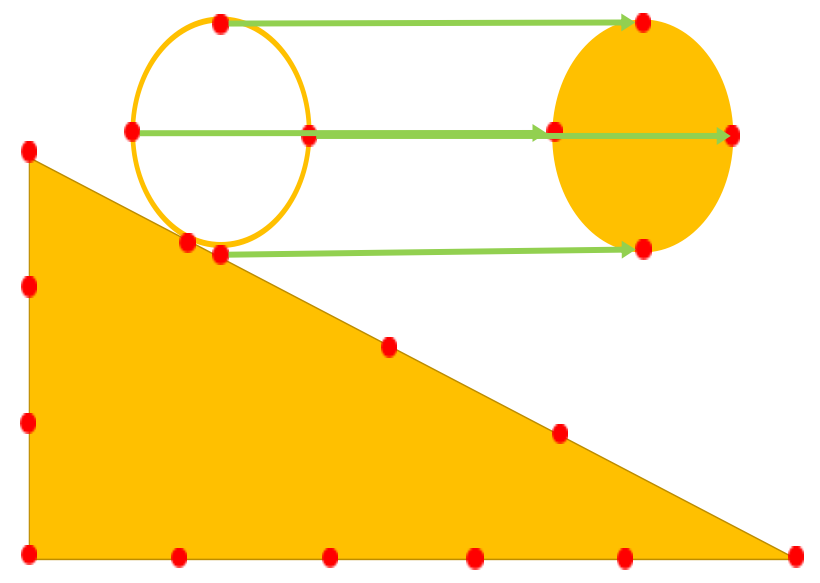

(c) Calculate the point moved

Fig.4. Future point 


\section{Driving Support System}

\subsection{Constitution}

The development environment is shown below.

- Windows 10

- Arduino: Arduino MEGA 2560 R3, Ver.1.6.8

- Camera: Elecom ucam-dle-300T series

- Voice recognition: voice recognition launcher $\mathrm{F}$

- Open cv: Open cv 3.1.0

The system configuration diagram is shown in Fig.1. Overall view of the system shows in Fig.2. The microphone and camera are always activated, and the driver uses his voice to activate the system. The voice recognition uses free software "Voice recognition launcher F", and when the word "Winker" is recognized, the camera starts image processing. After determining the face direction in the top, bottom, left, and right, data based on the face direction is sent to Arduino. Arduino, which received the data last, is the mechanism to operate the system. The system flow is shown in Fig.3. These actions do using two servo motors. Also, processing face detections is described below.

Up...Start of the hazard, and stop it

Down...If you start by mistake, the system does operation stop

Left...Start the left blinker

Right...Start the right blinker

\subsection{Distinction of Face Direction}

Optical Flow of Open CV is used to recognize. Optical Flow is time take advantage of image columns, image velocity (speed of object + camera speed) it represented by the vectors obtained in. To use the direction in which the person in the image moved, camera determines as following conditions.

(1) The same brightness of moving back and forth.

(2) Image is smooth.

(3) Moving value is very slightly.

(4) Neighborhood Pixel does the same motion.

In this system, after obtaining the feature points in the animation, a vector is calculated as to how much the feature point moved. A feature point is a point where it is always present and can be clearly distinguished from other feature points. Feature points are shown in Fig.4. Let the point which has the feature of Fig.4 be a red point. First, find feature points in the image. That is Fig.4 (a). And it is Fig.4 (b) that searched for the feature point of the image after moving. Finally, the vectors of feature points between the images are

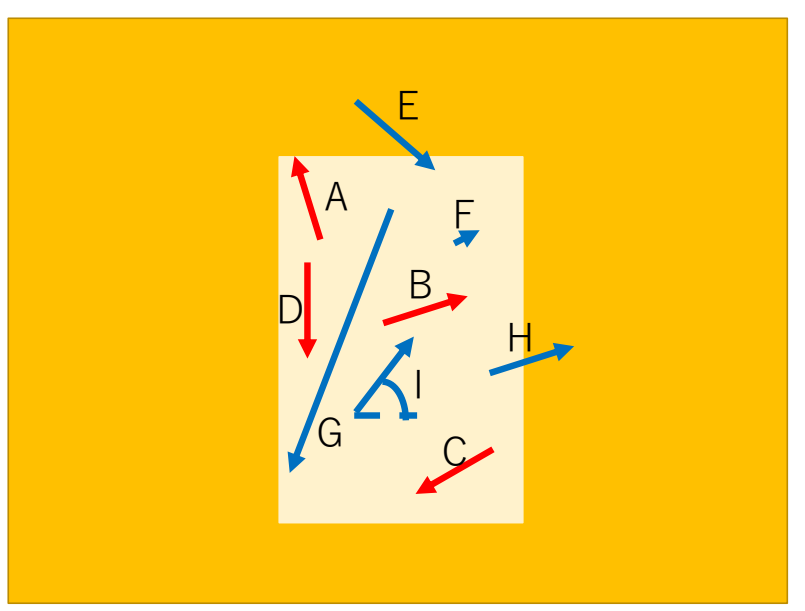

Fig.5. recognition condition of vector (1)

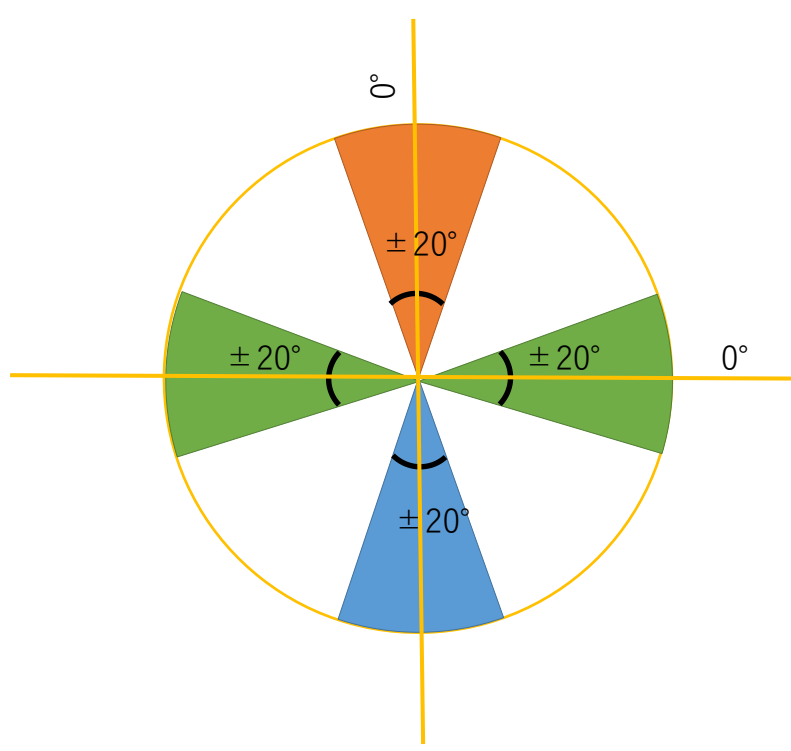

Fig.6. recognition condition of vector (2)

calculated. Also, the image size is $640 \times 480$ pixel. More of 220 Pixel in left and right at a time, on 80 Pixel, under 100 to calculate the vector in the range narrowed pixels. This is because the range in which the driver's face appears on the installed camera is this range. The vector calculated at this time has the minimum condition that both the start point and the end point are within the range. Also, too big vector is false recognition of feature point, too small vector is noise, so, to specify the range of the length of the vector. The vectors A, B, C, D satisfy all the conditions. The vector $\mathrm{E}$ is invalid because the start point is out of range. Vector $F$ is 


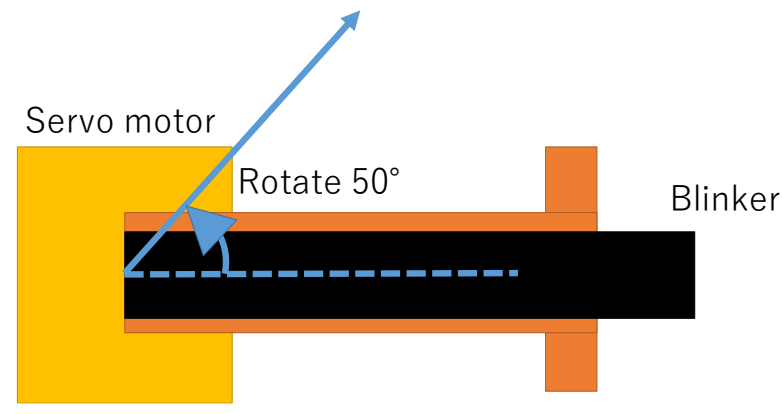

(a) initial position of blinker

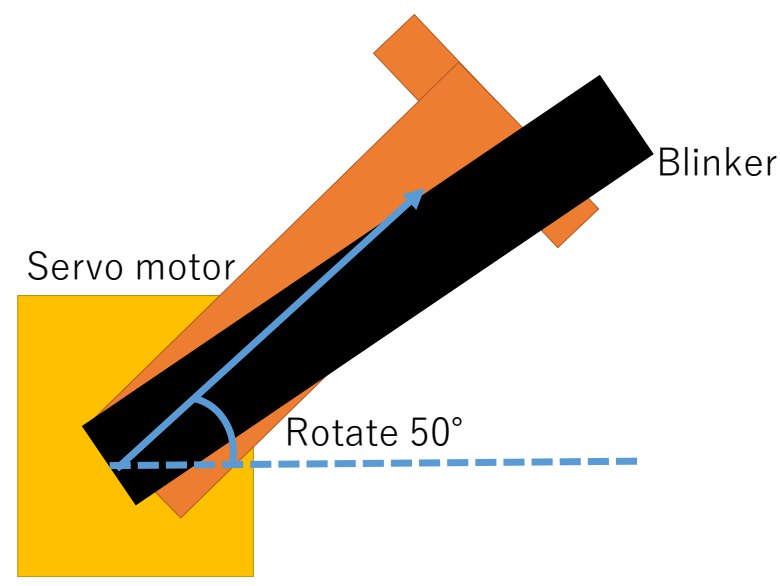

(b) start blinker

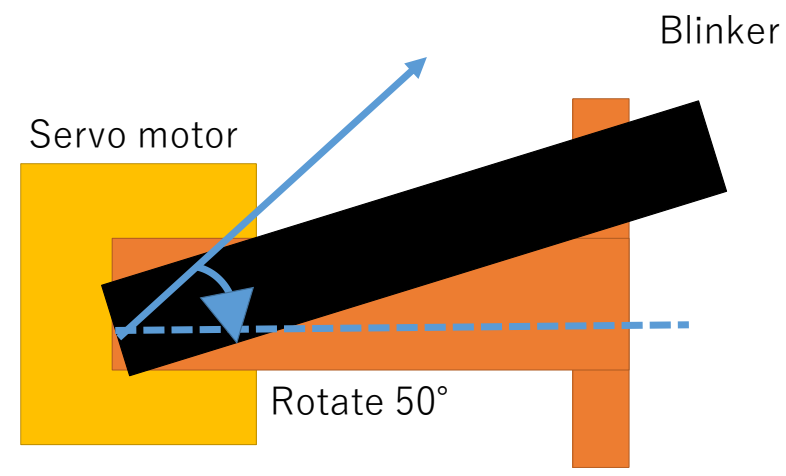

(c) servo return to initial position

Fig.7. motion part of blinker

invalid because it is too short. Vector $\mathrm{G}$ is invalid because it is too long. The vector $\mathrm{H}$ is invalid because the end point is out of range. Vector I is invalid because the angle is out of range. The distinction of left and right condition for $0^{\circ}$ line relative $\pm 20^{\circ}$ range of conditions. The upper and lower discrimination condition for a line rotate $90^{\circ} \mathrm{left} / \mathrm{right}$ distinction, it as $\pm 20^{\circ}$ range of conditions. These conditions are shown Fig.5, Fig.6.

\subsection{Motion Part}

The motion part of blinker is shown in Fig.7, the motion part of hazard is shown in Fig.8.

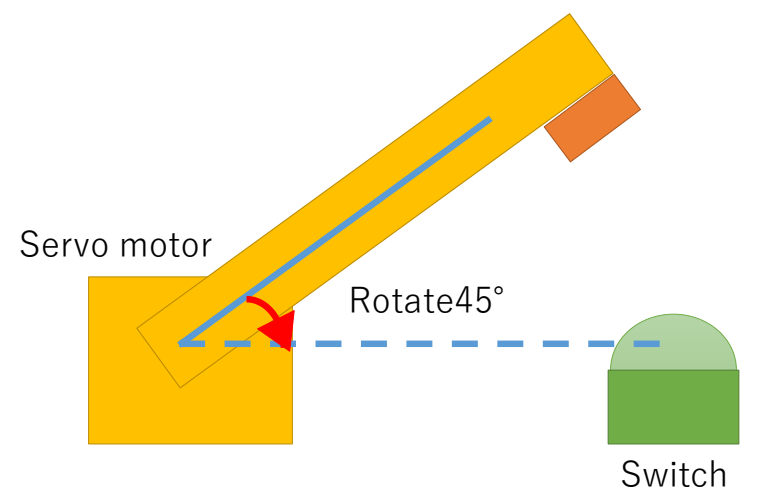

(a) servo rotate $45^{\circ}$

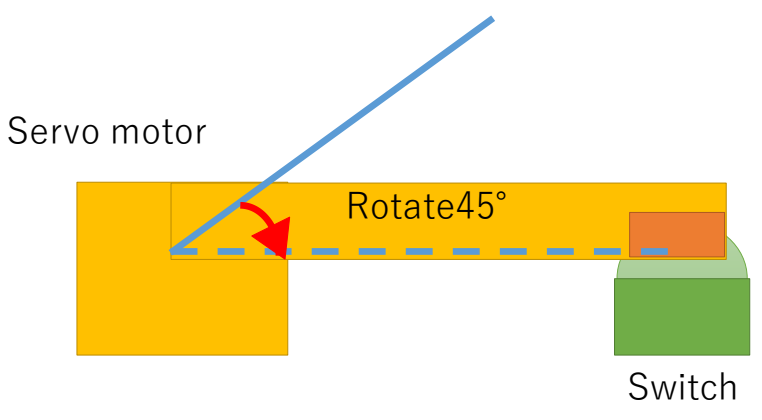

(b) Start hazard lamp

Fig.8. motion part of the hazard lamp

If the face direction is judged right by image processing, Arduino drives the servo in Fig.7. As linear position in Fig.7 rotate $50^{\circ}$ left and turn right signal flashing. And then, the servo motor rotates to the right $50^{\circ}$, and back to the original line. Because of this motion, the system can be applied to make the blinker turned off automatically when you turn the wheel back to its original position.

Next, if the face direction is judged left by image processing, Arduino the servo in Fig. 7 drive. Like the right signal, fig. 7 in relation to the position. From there the servo motor is rotated $50^{\circ}$ right to the left signal flashing. And then, the servo motor rotate to the left $50^{\circ}$.

Then, if the face direction is judged up by image processing, Arduino the servo in Fig. 8 drive. This is the contrivance that switch is pushed by the servo motor $45^{\circ}$ rotation. Besides, if the hazard lights are activated, camera can read the face direction only up. This makes, the hazard lights turn off.

In the end, if the face direction is judged down by image processing, Arduino the servo in Fig.7 drive and rotate $20^{\circ}$ both right and left. Because of this motion, you can stop the system when you start camera accidentally or blinker don't return to the initial position. 


\section{Experiment}

In this study, we identified the face direction from up, down, left, and right from voice recognition and confirmed the operation. The discrimination procedure of the face direction is shown in Fig.9, and the discrimination result of each direction is shown in Fig.10. The camera was installed

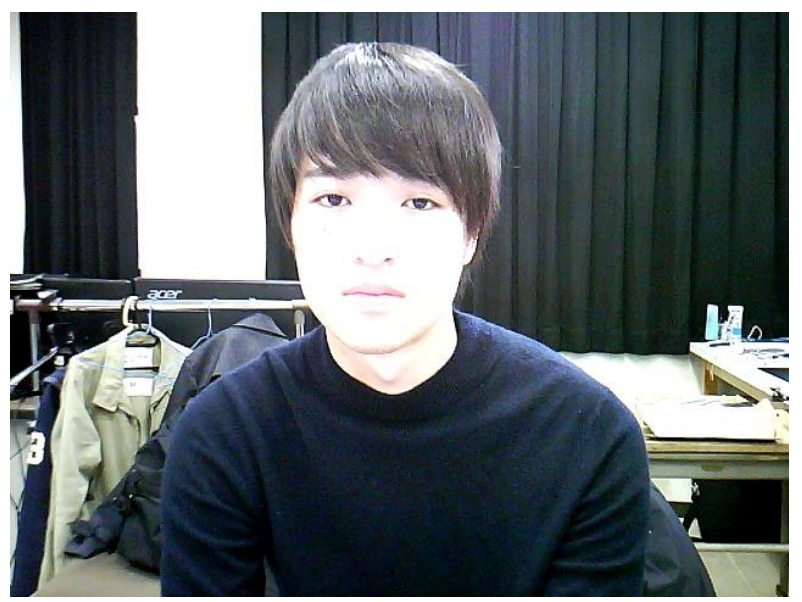

(a) Start the camera

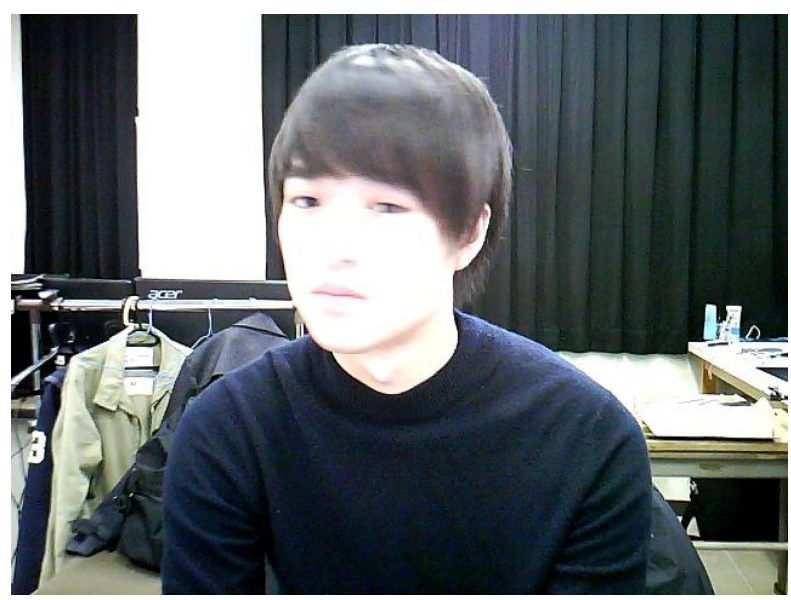

(b) Turn right

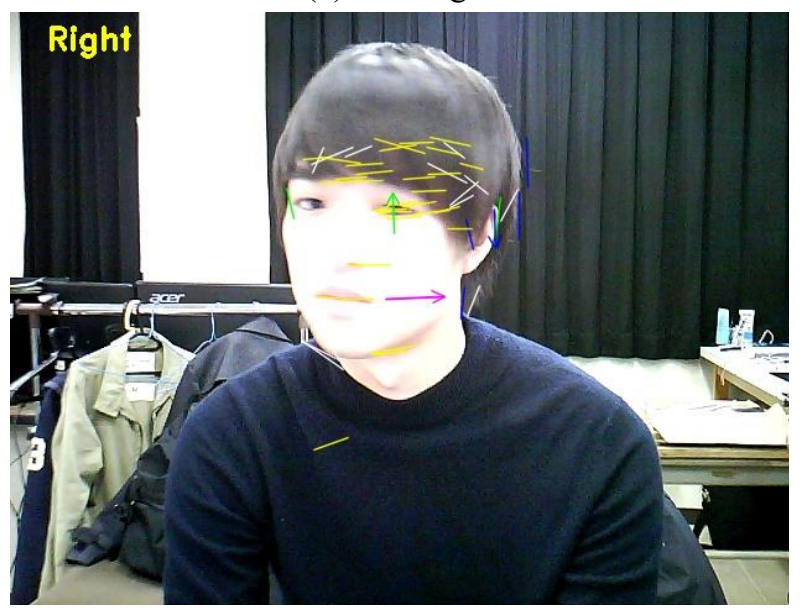

(c) Determine face direction

Fig.9. Face direction discrimination procedure

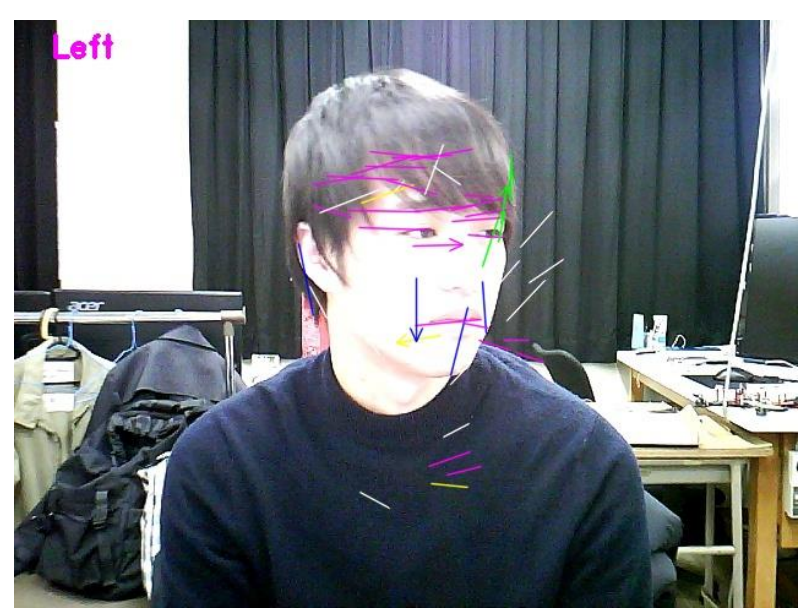

(a) Recognition of left

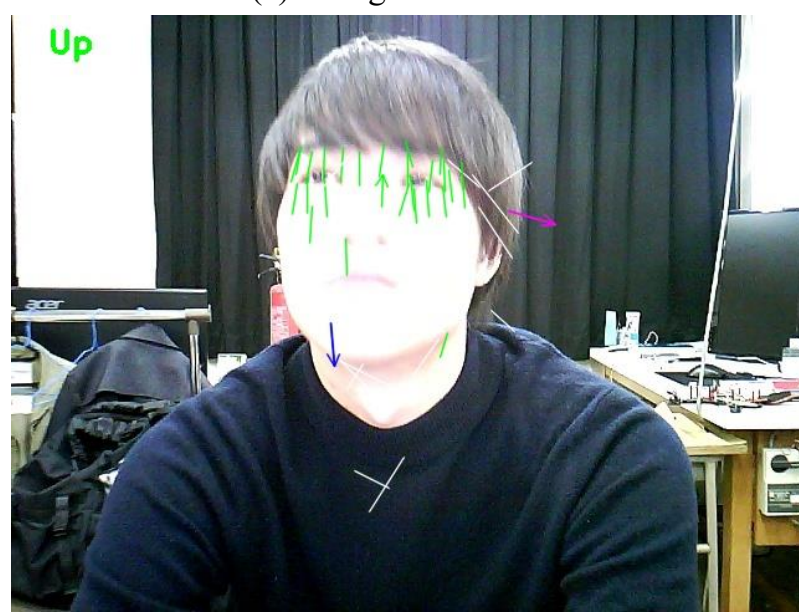

(b) Recognition of up

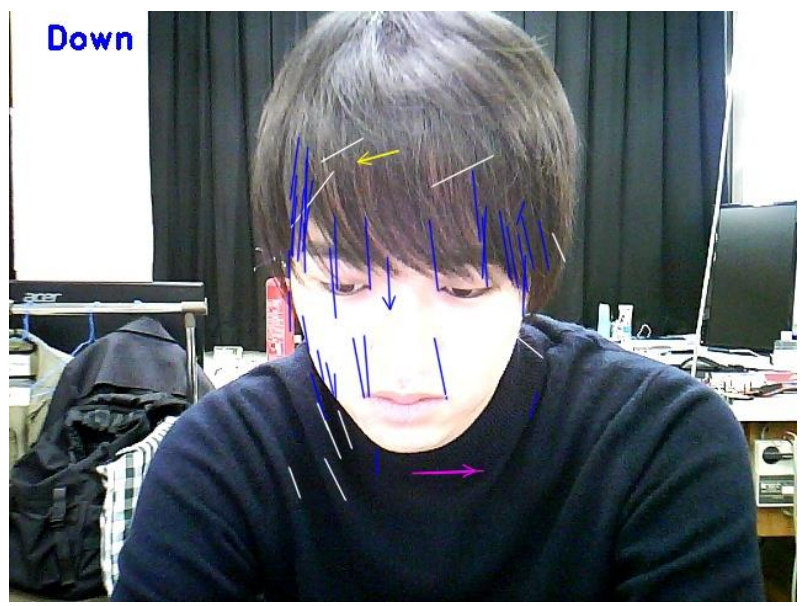

(c) Recognition of down

Fig.10. Face direction discrimination result

Table 1. Experimental result

\begin{tabular}{|c|c|c|c|c|}
\hline & Right & Left & Up & Down \\
\hline Success & 95 & 95 & 93 & 94 \\
\hline Failure & 5 & 5 & 7 & 6 \\
\hline $\begin{array}{c}\text { Success rate } \\
{[\%]}\end{array}$ & 95 & 95 & 93 & 94 \\
\hline
\end{tabular}


at a distance of $50 \mathrm{~cm}$ under fluorescent light, and the face direction was determined 100 times in each direction, and its performance was confirmed. The experimental results are shown in Table 1. As a cause of failure, if the moving speed of the face was too fast or if it was moving before the camera was activated, it was misrecognized. In other words, if the user moves the face after confirming the activation of the camera, false recognition is eliminated, so a person with a hand or fingertip obstacle can easily turn on the blinker or the hazard lamp.

\section{Conclusions}

In this research, we have developed a driving support system that can start and stop blinkers, activate hazards, and cancel motions from speech recognition. Moreover, in this experiment, we proposed in indoor environment. However, when actual use is assumed, there is a possibility that the optical flow may be disturbed due to the change of the brightness due to the change of the traveling environment. Therefore, it is necessary to measure the illuminance. In addition, it is necessary to conduct experiments in an outdoor environment by using infrared light. As a future subject, it is a subject to change a method, such as discriminating a face direction by machine learning so that discrimination can be performed in sunlight.

\section{References}

(1) National census of transportation method by statistics Japan http//www.stat.go.jp/data/kokusei/2000/jutsu1/00/04.ht $\mathrm{ml}$

(2) Statistics of driving license by police headquarters in 2017 : https://www.npa.go.jp/toukei/menkyo/index.htm

(3) Yoshimura Tadashi and Saito Yukio and Higashihara Takanori : "Reseach on rehabilitation hand with finger position and grasping force control", Proceedings for The Japan Society for Precision Engineering, pp 77-78, 2009

(4) Kato Mayumi and Sato Jun : "Visual Interface from Uncalibrated Cameras for Uncalibrated Displays", IEICE TRANSACTIONS on Information and Systems, pp 1-6, 2000

(5) Kunimi Kazuya and Yamazaki Yoichi : "Direction Indicator for Elderly based on Face Direction by Kinect", Human-Agent Interaction Symposium 2014, pp 66-67, 2014 